\title{
EL EXCURSO SOBRE MARCELO EN LAS HISTORIAS DE POSIDONIO: CRÍTICA Y REINTERPRETACIÓN DE LOS FRAGMENTOS*
}

\author{
Miguel Ángel Rodríguez Horrillo \\ Universidad de Zaragoza \\ horrillo@unizar.es
}

\section{THE EXCURSUS ABOUT MARCELLUS IN POSIDONIUS' HISTORIES: CRITICISM AND REINTERPRETATION OF THE FRAGMENTS}

\begin{abstract}
RESUMEN: Algunos fragmentos de las Historias de Posidonio tratan la figura de Claudio Marcelo, que cae fuera del arco cronológico de la obra. Ello llevó a los editores a proponer la existencia de un excurso sobre este personaje, una propuesta siempre sometida a duda. Tratamos de avanzar en esa idea proponiendo una única ubicación para la mayoría de los fragmentos en el excurso sobre los cognomina latinos, y subrayando la diferencia entre la imagen posidoniana de Marcelo y la de Plutarco.
\end{abstract}

PALABRAS CLAVE: Posidonio, historiografía, Marcelo, Plutarco.

\begin{abstract}
Some fragments of Posidonius' Histories deal with Claudius Marcellus. The impossibility to locate this historic character in the Histories' timeframe compelled the editors to suggest the existence of the so-called Excursus about Marcellus, a theory that is always call into question. We conclude that the majority of these fragments could be located in the excursus about the latin cognomina, and that the differences between the posidonian image of Marcellus and the plutarchean should be stressed.
\end{abstract}

KEYWORDS: Posidonius, Historiography, Marcellus, Plutarch.

RECIBIDO: 24.11.2013. ACEPTADO: 29.04.2014

Posidonio es, todavía hoy, un autor escurridizo. La importancia prototípica que la gran crítica alemana le confirió hace que su influencia se presienta a lo largo de toda la Antigüedad, y que el estudioso tenga la sensación de hallar sus enseñanzas en multitud de pasajes. La consecuencia más clara y tangible de esta

* Estudio realizado en el marco que ofrece el proyecto FFI2011-27501 del Ministerio de Economía y Competitividad. 
inseguridad es que cada editor tiene su propio Posidonio: el número de fragmentos presenta una variación de edición a edición tan grande que cualquier lectura global es un ejercicio realmente complejo. ${ }^{1}$

Las Historias son un buen ejemplo de esa situación: su imagen para la crítica va desde la muy estricta postura de la edición de Edelstein-Kidd -que solo admite fragmentos transmitidos con referencia clara a la persona de Posidonio-, a la de Theiler, que prácticamente edita libros enteros de Diodoro, pasando por la edición de Jacoby, a medio camino entre las dos anteriores, y que pone en marcha un inteligente juego de interpretación para dar una visión lo más completa posible de la obra historiográfica de Posidonio, siempre con la sensación de que muchos pasajes, tras cuya literalidad pudiera encontrarse Posidonio, quedan fuera de la edición. ${ }^{2}$ Esta situación hace que la imagen que la crítica ha ido construyendo de la obra sea una suerte de fantasma en el recorrido del género historiográfico antiguo: ${ }^{3}$ Posidonio parece expandirse con naturaleza líquida en el espacio que transcurre entre la obra de Polibio y el cambio de era, y su aliento intelectual parece respirarse tras cada una de las líneas de cierta enjundia que la historiografía del momento nos ha legado.

Estos dos problemas - la inseguridad del texto y su interpretación-, en definitiva, las dos caras de una misma moneda, han de caminar juntos a la hora de abordar cualquier interpretación de la obra del de Apamea, máxime si lo que perseguimos es entender la significación de una sección de la obra y así avanzar en la comprensión de las Historias como un todo. Para ello, proponemos el examen de unos fragmentos de autoría segura, y que dieron lugar a la construcción de una sección -0 incluso una monografía entera ${ }^{4}$ relativa a la figura de Marco

${ }^{1}$ Sin ánimo de exhaustividad, resultan útiles para conocer la figura y obra de Posidonio los trabajos de Reinhardt (1921; 1926), y especialmente su artículo en la Pauly-Wissowa (Reinhardt 1954), que ofrece una visión más moderada y ajena a la polémica si se lo compara con los anteriores trabajos, si bien en todos estos estudios la obra histórica se valora de manera marginal. Especialmente interesante es -a pesar de que también se centra únicamente en la vertiente filosófica del autor que nos ocupa-, el estudio de Edelstein (1936), crítico con la tendencia que ve a Posidonio en multitud de lugares, un trabajo que sirve tanto de contrapunto a ese "panposidonianismo" de Reinhardt como de introducción a los criterios que mueven la edición de Edelstein-Kidd (1972). Para las Historias, además del comentario de Jacoby (1963), es fundamental el estudio de Malitz (1983), por el esfuerzo que supuso, a pesar de que su dependencia de pasajes diodoreos que solo en teoría dependen de Posidonio haga que hoy sea, en algunos aspectos, una obra de difícil manejo. En ámbitos hispanos puede verse el artículo de carácter general de Candau (1985: 116-125). Salvo indicación expresa, citamos los fragmentos por la edición de Jacoby.

${ }^{2}$ Vid. para estos problemas las indicaciones de Clarke (1999: 130-139), con un marcado escepticismo, reacción lógica ante la excesiva confianza de la crítica a la hora de reconstruir un autor prácticamente perdido por completo, pero que es fundamental para nuestro conocimiento de la Antigüedad.

${ }^{3}$ Pueden verse las razones aducidas e. g. por Ferrary (1988: 489, n. 7), para dejar prácticamente fuera de su estudio las Historias de Posidonio: los fragmentos seguros son demasiado pocos como para aventurarse en una reconstrucción profunda, que además siempre sería escasa si se la compara con las expectativas que la crítica se ha creado.

${ }^{4}$ Como fue la propuesta de Mühl (1925: passim y especialmente 35), aceptada con las debidas precauciones por Münzer (1925: 97) en su reseña a la obra de Mühl. 
Claudio Marcelo. La existencia de ese excurso, que ha sido puesta en tela de juicio por los estudiosos -especialmente por Kidd- ${ }^{5}$ fue uno de los puntos a partir de los cuales la crítica trató de conocer la interpretación que Posidonio hacía de Roma y su cultura: ${ }^{6}$ aunque la idea del excurso siempre haya tenido poca fuer$\mathrm{za}^{7}$ los planteamientos interpretativos derivados del mismo todavía están presentes en algunos razonamientos de la crítica posidoniana, sin que se deslinde de manera totalmente clara qué pertenece al pensamiento de Posidonio y qué a Plutarco, que es quien nos transmite los fragmentos en cuestión. Analizando estos aspectos podremos avanzar en la comprensión de la imagen que Posidonio tenía de la cultura romana, y su relación con el resto de los pueblos tratados en las Historias. Así, se hace necesario abordar un examen crítico y desprovisto de apriorismos de los fragmentos F41, 43, 44 y 60, a fin de verificar la realidad o no de esa construcción y, sobre todo, la posible imagen que Marcelo tendría en la obra de Posidonio, siempre interpretada -con o sin excurso- en términos positivos, visión que, a nuestro entender, ha de ser matizada.

\section{El supuesto EXCurso laudatorio sobre Marcelo}

Los fragmentos relativos a la figura de Marcelo son de manejo incómodo para el editor: por razones obvias de cronología, dado que los acontecimientos de la vida de Marcelo son anteriores al año en el que comienza la obra, no pueden insertarse sin más en el continuo de la narración histórica, motivo por el cual la crítica propuso la existencia de un excurso dentro de las Historias de Posidonio, ${ }^{8}$ cuya localización es una cuestión realmente complicada, por no hablar, como indicábamos, de la propuesta de una obra monográfica relativa a Marcelo. El único punto de apoyo es la supuesta cercanía de Posidonio con el Marcelo cónsul del año 51 a. C., 9 y los lazos de amistad con esta familia, lo que podría dar respuesta

${ }^{5}$ Puede verse una revisión de los argumentos en Kidd (1988: 345-346).

${ }^{6}$ Cf. e. g. el examen de Ferrary, que une estos pasajes con el importante núcleo que constituyen los libros 33-37 de Diodoro (Ferrary 1988: 493-494), o el reciente tratamiento de Clarke (1999: 180-181).

${ }^{7}$ Sin embargo, todavía pueden leerse observaciones algo confusas al respecto (e. g. Dale 1992: 23).

${ }^{8}$ Dejamos a un lado el tratamiento pionero, pero insuficiente, de Bake (1810: 171-173), quien se limitaba a recoger los fragmentos y a ofrecer una suerte de paráfrasis de los mismos. Cf. para la idea del excurso las dudas de Müller (1849: 231-232), quien se debatía ya en fecha tan temprana entre otro escrito del de Apamea o algún excurso en la obra historiográfica (Cf. también Müller 1849: 270).

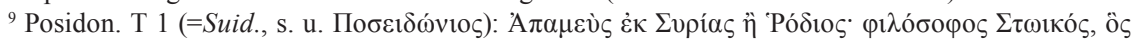

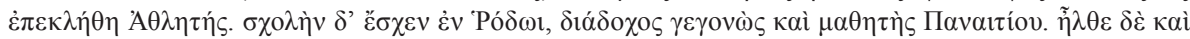

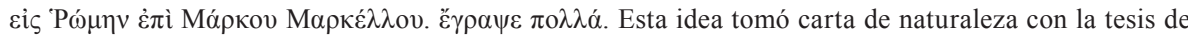
Toepelmann (1867: 39): ...ita ut amicitia, quae Posidonio cum hoc Marcello, Pompeii socio [...] intercedebat, ansa nobis sit ad recte de fragmentis illis iudicandum. Ha de notarse que el propio autor (Toepelmann 1867: 2), creía que decidir si estamos ante una obra independiente sobre Marcelo o, simplemente, ante un excurso, es algo que depende de si Plutarco usó a Posidonio como fuente fundamental para su biografía, cosa que hoy sabemos que no fue así. Para la reconstrucción de la vida de Posidonio pueden verse los datos presentados por Reinhardt (1954: 563-564): nótese cómo Reinhardt cae en un argumento circular al defender la existencia de una vieja amistad de Posidonio con los Marcelos a partir de la fecha 
al origen del excurso - entendido como un derivado literario de esa amistad-pero no a la ubicación del mismo en la obra. Por desgracia, Plutarco, quien nos proporciona los fragmentos en cuestión, nos ha transmitido un material lo suficientemente limitado como para no poder determinar con profundidad su significación, pero, a pesar de ello, algún indicio se puede obtener de los mismos. Peter ya indicó de manera clara que el uso que Plutarco hizo de Posidonio como fuente para su biografía daba la impresión de que el material proporcionado por este último no permitía dar una visión cabal y completa de los acontecimientos de la vida de Marcelo, sino dar cuenta únicamente de ciertas anécdotas (Peter 1865: 77). ${ }^{10}$ A esta naturaleza fragmentaria de la fuente posidoniana se ha de unir el hecho de que Marcelo fue objeto de idealización o de manipulación muy pronto (Münzer 1899: 2750-2751):: Celio Antípatro conocía tres versiones de su muerte, ${ }^{12}$ y la versión de Polibio era realmente dura con la actuación del personaje en el momento de su muerte, tachada directamente de imprudente (Pol. 10.32). ${ }^{13}$ Surge así una tradición negativa que contrastará con las versiones más dulces de Tito Livio y Plutarco, y es en este contexto en el que tenemos que situar los fragmentos posidonianos que, si atendemos a los motivos de amistad con los Marcelos que habrían llevado a Posidonio a redactar el excurso, habrían de ser necesariamente positivos, ${ }^{14}$ incluso aunque no admitamos la existencia de un excurso como tal. ${ }^{15}$

del viaje, la renovación del acuerdo entre Rodas y Roma, y la existencia del excurso. En este mismo sentido se expresa Pohlenz (2005: 424, n. 6). Laffranque (1964: 150), presenta un examen muy escaso de la sección que nos ocupa, algo lógico dentro de un tratamiento general de la figura del de Apamea.

${ }^{10}$ Cf. también de Sanctis (1968: 368), y Dale (1991: 21-24). En contra de esta propuesta pueden verse las indicaciones de Wilamowitz (1955: 397, n. 1), y Mühl (1925: passim); la opción de tomar todo aquello que tuviera tinte estoico en la biografía plutarquea como posidoniano es la causa de que Mühl proponga la existencia de una monografía del de Apamea sobre Marcelo. Para valorar este exceso, puede apuntarse simplemente que este crítico (1925: 6), toma el adjetivo $\sigma \omega ́ \varphi \rho \omega v$ como indicio de la naturaleza posidoniana de un pasaje.

${ }^{11}$ Como bien señala Caltabiano (1975: 64), apuntar una cronología aproximada en ese proceso de manipulación es simplemente imposible.

${ }^{12}$ Para una recopilación y examen del material disponible sobre la vida de Marcelo, puede verse el estudio de Flower (2003: 34-52). Sobre los pormenores del fragmento 29 Peter baste remitir al comentario de Herrmann (1979: 154-167), especialmente para el estudio de las relaciones entre el texto de Celio Antípatro y el de Tito Livio, de donde procede el fragmento. Los pasajes están recogidos en el artículo de Münzer (1899: 2754).

${ }^{13}$ Para el pasaje polibiano referido y las fuentes sobre la muerte de Marcelo, baste recurrir a las notas del comentario de Walbank (1967: 242-243).

${ }^{14}$ Para la manipulación de la tradición histórica por parte de los Marcelos, pueden verse e. g. los datos presentados en el estudio de Flower (1996: 147).

${ }^{15}$ Cf. para esta idea los comentarios de Jacoby (1963: 189) y Kidd (1988: 346): "Posidonius, because of the Marcellan connection with Rhodes (F258), or because of his personal acquaintance with contemporary leading members of the family (258 comm.), may have derived from them information about their famous ancestors, [...] and may even have attempted to correct in passing the more hostile tradition against them stemming from Polybius (F271 Komm.)." Cf. también la reconstrucción de Malitz, (1983: 362), quien llega a proponer que detrás de algunas secciones de la vida plutarquea hay más pasajes del excurso posidoniano, cosa que entra en colisión con la opinión sostenida por la crítica desde el estudio de Peter (supra, nota 8), y supone suavizar la postura de Mühl. 
Es precisamente esta cuestión del sentido de los fragmentos lo que habrá de ser valorado con especial atención.

Por si fueran pocos estos problemas, a nuestro entender, estos fragmentos posidonianos han sido tratados de una manera inconexa y no como un único núcleo con sentido propio. Es esta una visión propiciada por su dispersión por la vida plutarquea y por esa necesidad de generar material diferente y diferenciado para el excurso, visión mantenida incluso por quienes no defendían la existencia de la sección relativa a Marcelo. Sin embargo, el sentido de estos pasajes y la acumulación en ellos de datos referidos a este personaje permiten plantear que el supuesto excurso sobre Marcelo podría enmascarar una vinculación de esos fragmentos, aunque no se tratara de un excurso referido a Marcelo: estaríamos hablando de otro excurso, que no estaría centrado en este personaje, pero en el que se emplearía a Marcelo como ejemplo fundamental. Obviamente, nos estamos refiriendo al núcleo que supone el análisis de los cognomina en la obra de Posidonio.

Para justificar esta afirmación, comenzaremos por analizar en bloque los fragmentos F41 y F44, exactamente el comienzo y el final de la vida plutarquea. En el primero de ellos, Plutarco recoge la etimología posidoniana del nombre de Marcelo, seguida de una descripción del carácter del personaje introducida por $\gamma \alpha$ ó $\rho$. A nuestro entender, la segmentación del fragmento por parte de Jacoby puede dar lugar a confusión: el texto termina con unos puntos suspensivos que dejan al lector con la duda de lo que sigue en el texto plutarqueo. ${ }^{16}$ Todo el pasaje dice lo siguiente:

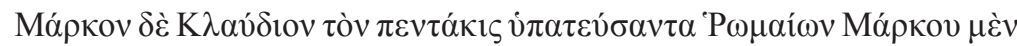

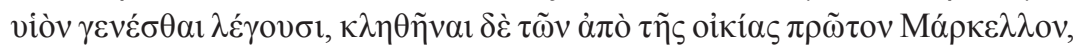

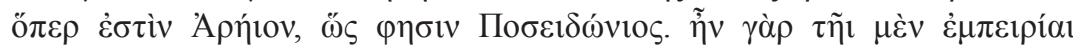

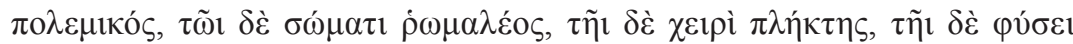

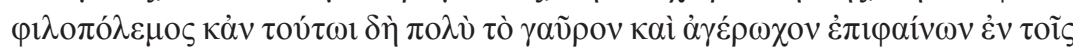

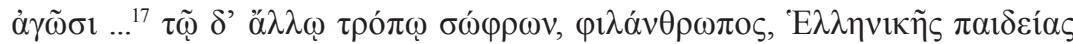

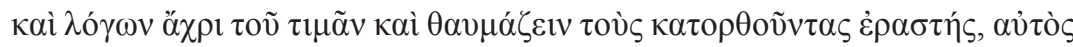

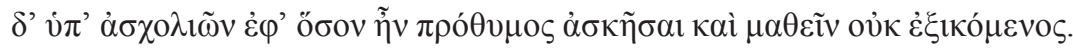

El lector atento habrá observado la clara cesura que se abre entre la primera parte de la caracterización del personaje y la segunda, iniciada con la expresión $\tau \tilde{\omega} \delta^{\prime}{ }^{\prime} \not \partial \lambda \lambda \omega \tau \rho o ́ \pi \omega$ y que prácticamente entra en contradicción con la primera sección del texto, aquella que dependía tan marcadamente de la etimología gracias al conector causal. Kidd, con su habitual escepticismo, editó todo el texto que presentamos, pero tuvo la precaución de indicar que solo la primera

\footnotetext{
${ }^{16}$ Jacoby recorta intencionadamente el texto que había editado Müller, que abarcaba todo el primer capítulo de la vida plutarquea (Müller 1849: 270).

${ }^{17}$ Hasta aquí el fragmento tal y como lo presenta Jacoby. Kidd edita la totalidad del texto que presentamos, eliminando los puntos suspensivos que cerraban el fragmento tal y como lo recogía Jacoby.
} 
frase era posidoniana: el resto podía serlo también, al menos el estilo así lo indicaba, pero la actuación sobre el texto por parte de Plutarco era algo con lo que había que contar (Kidd 1988: 902). ${ }^{18}$ A nuestro entender, esta complejidad se resuelve si se presta atención al contenido de cada una de esas dos partes en que se divide el texto. La primera de ellas nos dibuja un personaje cuasi homérico -no en vano la crítica ha llamado la atención sobre el término ả $\gamma \varepsilon$ $\rho \omega \chi 0 \varsigma,{ }^{19}$ de fuerte peso homérico y de sentido negativo en Plutarco $-{ }^{20}$ mientras que la segunda nos

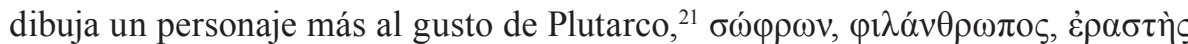
'E $\lambda \lambda \eta v i \kappa \eta ̃ \varsigma ~ \pi \alpha 1 \delta \varepsilon i ́ \alpha \varsigma$. El estudio de Martin sobre la filantropía como valor en las biografías plutarqueas es definitivo en este sentido (Martin 1961: 172): ${ }^{22}$ Marcelo es caracterizado como tal a lo largo de toda la vida, por lo que esa caracterización -y con ella, creemos, los restantes rasgos de la segunda parte- se debe a Plutarco, quien habría regularizado el material de la biografía bajo ese principio.

La deducción lógica es clara: la primera mitad del texto, sin los puntos suspensivos de Jacoby y con un punto final si se quiere, es el material tomado de las Historias de Posidonio, incluida la etimología, en tanto que la segunda parte es

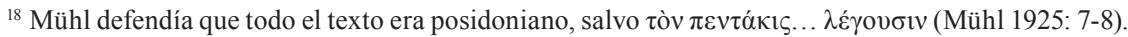

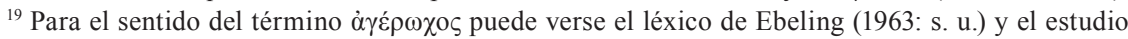
de Buttmann (1861: 18-20). El sentido es claramente positivo en la tradición homérica, siempre referido a guerreros y en un contexto -en términos modernos- de brutalidad guerrera, pero negativo por ello mismo en autores posteriores, como por ejemplo en el inmediato predecesor del de Apamea, Polibio, para el cual puede verse también el léxico de Mauersberger (1968: s. u.) En general, para el empleo de Homero por parte de Posidonio, pueden verse las indicaciones de Rudberg (1918: 26-27 y 127-128).

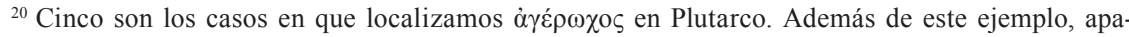
rece en una referencia a Homero en Plu. Fab. 19.2, un pasaje gemelo del que nos ocupa -no en vano es el fragmento F42b, aunque en Jacoby esté cortado-y que confirma el tono homérico del concepto a ojos

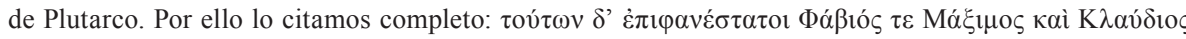

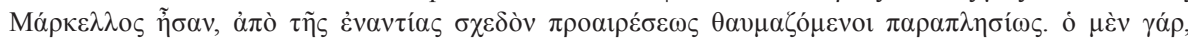

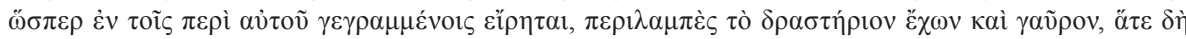

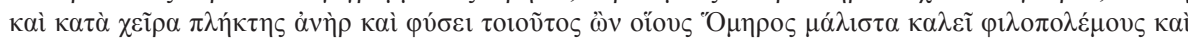

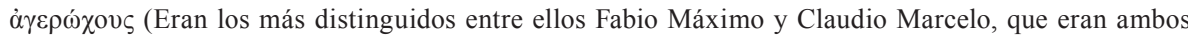
igualmente admirados, aunque por su línea de conducta casi totalmente opuestos. Aquél, como se ha dicho en su Vida, brillaba por su actividad y orgullo, y era además un hombre de brazo fiero y de natural comparable a los que Homero llama sobre todo amigos de guerra y arrogantes." (Trad. A. Pérez Ji-

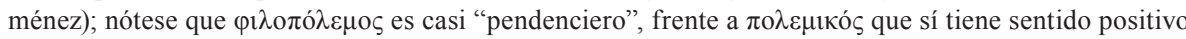

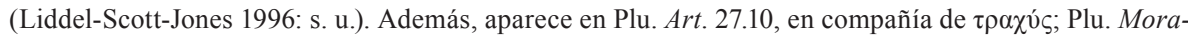

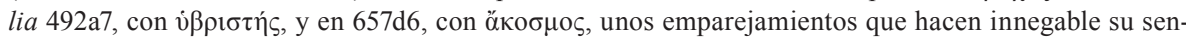
tido negativo a ojos de Plutarco.

${ }^{21}$ Cf. sobre estos pormenores el comentario de Theiler (1982: 89). Nótese que, en la vida plutarquea, el de Queronea hace seguir a esa descripción virtuosa de Marcelo una referencia homérica, por lo que la continuidad del texto se mantiene, y el lector pasa de una a otra sección casi sin darse cuenta. La filantropía es en Plutarco casi un sinónimo del concepto de civismo en términos griegos (Becchi 2009: 268).

${ }^{22}$ Para estos pasajes y, en líneas generales, para la asignación de este concepto a Plutarco y no a sus fuentes, baste remitir al mismo trabajo (Martin 1961: 165), y para el concepto de filantropía en el universo plutarqueo, a los recientes trabajos del volumen que recoge los estudios presentados al Congreso de la Sociedad internacional de plutarquistas celebrado en 2008 en Coimbra (Ribeiro Ferreira, Leâo, Tröster y Barata Dias 2009). 
un añadido debido a Plutarco, ${ }^{23}$ quien seguramente quiso hacer uso del material etimológico y no pudo eliminar la explicación del mismo, a pesar de que gene-

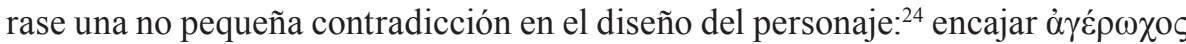
con $\sigma \omega ́ \varphi \rho \omega v$ es algo, a nuestro entender, difícil, dado que ả $\gamma \varepsilon \dot{\varepsilon} \rho \omega \chi \varsigma$ tiene siempre un sentido negativo en Plutarco, incompatible con $\sigma \omega ́ \varphi \rho \omega v$, hasta el punto de que en todos los casos acompaña a adjetivos cuyo significado es contrario al de este último término.

Una vez aislada la sección posidoniana en este pasaje, podemos abordar el fragmento F44, cuyo núcleo es el epigrama que adornaba la estatua de Marcelo en Lindos:

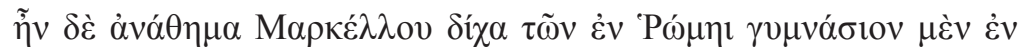

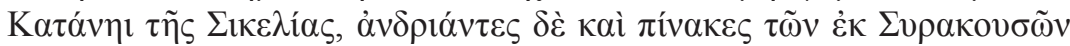

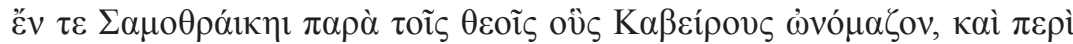

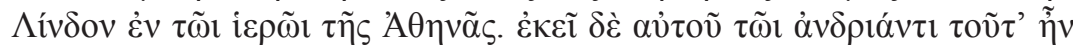

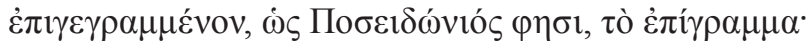

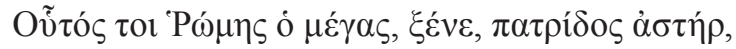

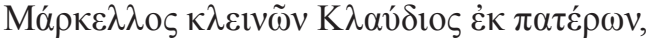

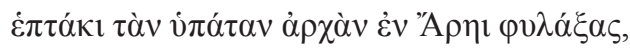

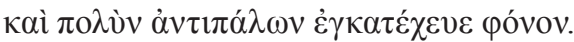

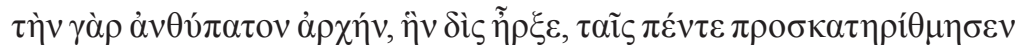

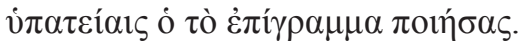

El texto es, como mínimo, bastante sospechoso: Klotz atribuía su redacción a alguien no muy versado en la vida de Marcelo, dado que las siete veces que ejerció el consulado no tienen encaje posible con la realidad histórica (Klotz 1934: 293),

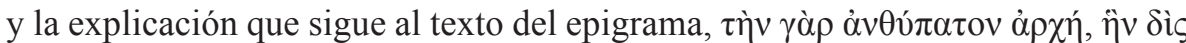

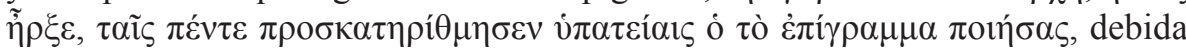
con casi total seguridad a Plutarco, ${ }^{25}$ no permite explicar ese error. ${ }^{26}$ Pero los problemas no acaban aquí: la lectura de la primera línea fue corregida por Stephanus

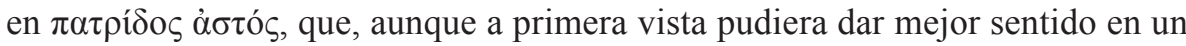

${ }^{23}$ Para la modificación y adaptación de las fuentes por parte de Plutarco vid. el trabajo clásico de Pelling (1980: 126-131).

${ }^{24}$ Contradicción que no parece advertir Ferrary (1988: 493-494, n. 24), si bien en su tratamiento de los fragmentos no suele aislar al transmisor y al transmitido, como ocurre, por ejemplo, en su análisis del F43 (Ferrary 1988: 509): en ello se evidencia el manejo de la edición de Theiler que, como decimos, es especialmente insensible a estos aspectos.

${ }^{25}$ Las dudas de Kidd (1988: 900) en lo que se refiere la atribución del razonamiento a Plutarco nos parecen excesivas. Más firme se muestra Dale en su comentario (Dale 1992: 375).

${ }^{26}$ Recuérdese que Marcelo ejerció el proconsulado, como advertían Klotz (1934: 293) y Dale (1992: 375), cuatro veces. 
contexto referido a un personaje romano, ${ }^{27}$ plantea no pocos problemas dado el significado exacto del término, vinculado con derechos civiles pero no políticos. Más complicado es el caso del tercer verso en su segunda mitad, غ̇ $\pi \tau$ ć́к।

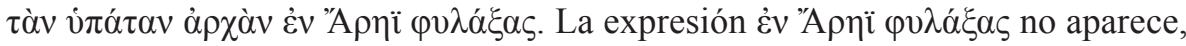
tras una búsqueda informática, en ningún otro pasaje conservado de la literatura griega, y a esta complicación en lo que se supone que tiene que ser un texto poético cargado de reminiscencias, se une el muy difícil último verso, en el que los problemas textuales se verifican en kaí, que aporta poco sentido en la construcción de la sintaxis del texto - un anacoluto difícilmente permisible en un texto tan corto y sencillo-y en $\dot{\varepsilon} \gamma \kappa \alpha \tau \dot{\varepsilon} \chi \varepsilon v \varepsilon$, solo localizado en el presente texto. ${ }^{28}$

A nuestro entender, los problemas son lo suficientemente numerosos -incluso en lo referente al contenido-, como para pensar que la corrupción del epigrama se haya producido en el curso de la transmisión del texto..$^{29} \mathrm{El}$ epigrama está, seguramente desde su composición, aquejado de esos defectos que la crítica ha señalado, y nos pone ante un problema no menor: no se trata simplemente de que sea un epigrama con errores en su contenido, sino que seguramente es así como Posidonio lo insertó en su obra. De esta circunstancia se derivan dos consecuencias: por una parte, se refuerza la vinculación que creemos que existe entre el poema y el fragmento anterior relativo a la etimología, dado que en ambos aparece la referencia a Ares, y por otra, la idea de esa cercanía de Posidonio con los Marcelos se complica, dado que es difícil justificar la existencia en el de Apamea de un error histórico relativo a la carrera de Marcelo si esa cercanía fuera tan marcada. De lo contrario, lo lógico es pensar que habría corregido el epigrama a la luz de la información de los archivos de la familia.

Respecto a la primera consecuencia, $\operatorname{Kidd}^{30}$ propuso que Posidonio tomó

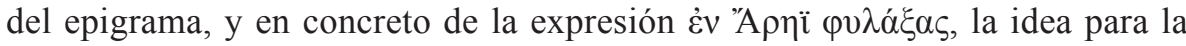
etimología del nombre de Marcelo. Esta propuesta puede plantear dudas, pero nos abre la puerta, como decimos, a la vinculación de los dos fragmentos, que a nuestro entender formarían parte de un mismo segmento de la obra de Posidonio. La secuencia de los textos se aclara si asumimos que a la presentación de la etimología le seguía la caracterización del personaje con los datos de la

\footnotetext{
${ }^{27}$ Ziegler da por buena esta lectura y la admite en su edición de la vida plutarquea (Ziegler 1935: 63, y Ziegler 1994).

${ }^{28} \mathrm{Cf}$. la corrección de $\kappa \alpha$ é en la edición de Ziegler, y la retractación de Gärtner en los addenda (Ziegler 1994: 343), ajustando así la edición de Ziegler a la de Flacelière y Chambry, para lo cual vid. nota siguiente.

${ }^{29}$ Ziegler hablaba del epigrama como de una "chapuza" en los dos últimos versos (Ziegler 1935: 64). La edición más reciente de Flacelière y Chambry no supone avance alguno, dado que ofrece un texto muy conservador, pasando de puntillas por el problema: salvo las indicaciones en el aparato crítico, no tenemos nota alguna por parte de los editores (Flacelière y Chambry 1966: 232).

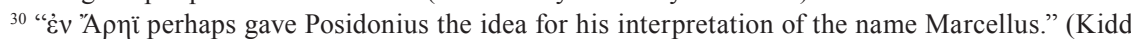
1988: 900).
} 
descripción posidoniana, y a ello, a modo de ejemplificación, el epigrama recogido por Plutarco.

De este modo, los dos fragmentos constituyen, en esencia, un único núcleo de la obra posidoniana, lo que permite aclarar un poco más la realidad o no del supuesto excurso sobre Marcelo. Jacoby apuntaba ${ }^{31}$ que el fragmento F41 pertenecía a ese supuesto excurso sobre la figura de Marcelo y no al pasaje en el que Posidonio analizaba el sistema onomástico latino. La primera contradicción en esta afirmación ${ }^{32}$ se puede observar en el propio texto editado por Jacoby, quien nos remite en el fragmento F60 -referido al sistema onomástico latinoal fragmento F41, y el editor hace la referencia al pasaje que nos ocupa precisamente cuando se habla de Marcelo en el análisis de los cognomina. Si se sigue con atención el razonamiento, Plutarco nos deja entrever el porqué de que Posidonio atribuyera esa importancia al tercer nombre: era el que caracterizaba de manera individual a su posesor, como ocurría con el Africano o Metelo Macedónico. ${ }^{33}$ En términos de pensamiento filosófico, la comprensión del sistema onomástico se ha de realizar no desde nuestro conocimiento técnico del mismo, ${ }^{34}$ sino desde la percepción de un pensador más preocupado por la esencia característica de los individuos que por datos de otra índole. Desde esta óptica se comprende el fácil encaje que el conglomerado formado por los fragmentos F41 y F44 tiene con el fragmento F60: los dos primeros fragmentos serían una de las posibles ejemplificaciones de la naturaleza del cognomen, como también lo sería la contraposición entre Marcelo y Fabio Máximo que conocemos por dos contextos plutarqueos (=Posidon. F42a-42b), y que se refiere también al carácter belicoso de Marcelo.

De este modo, todos estos fragmentos serían parte de la explicación del cognomen en un caso relativamente más complejo a primera vista que los de "el Africano" o "el Macedónico", mucho más simples. En este sentido, la inercia general de la obra de Posidonio hace que, como se indicó por la crítica, se observe una equiparación de estos nombres con el paralelo oriental de epítetos referidos a la caracterización del individuo, aspecto que vemos claramente en el caso de

\footnotetext{
31 "Doch stammt die Charakteristik nicht aus dieser Erklärung des Beinamens enthielt, sondern aus dem Marcellusexkurs." (Jacoby 1963: 190).

${ }^{32}$ Esta ambigüedad seguramente llevó a Malitz a considerar que el fragmento podría tener una de las dos ubicaciones (Malitz 1983: 363).

${ }^{33} \mathrm{Cf}$. el reciente tratamiento de van Nuffelen, quien distingue un cambio en el uso de los epítetos por parte de los historiadores precisamente de Polibio en adelante, materializado en su uso como nombre de los monarcas orientales y en una mayor preocupación por la explicación de los mismos (van Nuffelen 2009: 95-107).

${ }^{34}$ Cf. para todo ello el estudio de Schulze (1991: 506-507). Para una indagación de las complejidades derivadas de la importancia dada por Posidonio al cognomen y las dificultades de la posterior reflexión de Plutarco, puede verse el comentario del editor inglés (Kidd 1988: 907-908). A este respecto, resulta esclarecedor el capítulo de Balsdon sobre este tema (Baldson 1979: 148-149).
} 
Ptolomeo VIII. ${ }^{35}$ Esta explicación tiene además buen anclaje en esa preocupación por los aspectos psicológicos y antropológicos ${ }^{36}$ que recorre la obra de Posidonio y que le dan esa naturaleza tan característica: Klaus Bringmann señaló (Bringmann 1985: 30) ${ }^{37}$ la importancia de estos aspectos como un factor unificador de primer orden de la obra posidoniana, en el marco seguramente de aquella famosa afirmación de Ateneo respecto al pulso filosófico con el que Posidonio redactó su obra. ${ }^{38}$

Finalmente, resulta mucho más complejo tomar cumplida cuenta de los efectos de esos errores de contenido del epigrama en la comprensión de la posición de Marcelo en la obra de Posidonio. La postura unánime de la crítica apunta a una relación directa y firme entre Posidonio y la familia de Marcelo (Vimercati 2004: 678 , con bibliografía anterior), pero parece difícil que la materialización de esa relación y del uso de los archivos familiares sea dar cabida en su obra histórica a un epigrama cuyo contenido es simplemente falso, y hacerlo sin indicaciones claras de esas dificultades, por no hablar del apelativo de arrogante. Este fragmento, y con él el relativo a la etimología del nombre, difícilmente pueden formar parte de un tratamiento laudatorio de la figura de Marcelo, y abren un escenario que agrava si cabe las dudas respecto a la existencia del excurso, y que plantea también problemas respecto al tono laudatorio de la visión de Marcelo en las Historias.

Queda únicamente por examinar el fragmento F43, que la crítica alemana puso ya en cuarentena en lo que a la interpretación propuesta por Jacoby se refiere, y que estuvo en el origen de la asignación de una nueva obra a Posidonio, la ya aludida monografía sobre Marcelo. Sin embargo, esta postura se asienta sobre un manejo del pasaje - una vez más transmitido por Plutarco-que se nos hace difícil de sostener, habida cuenta de los avances en nuestro conocimiento del modo de trabajar de Plutarco. ${ }^{39}$

La comprensión del fragmento, tal y como aparece en todas las ediciones, puede ser y ha sido objeto de una interpretación, dentro de ese supuesto excurso

${ }^{35}$ Cf. supra, nota 34. Para estas cuestiones vid. el artículo de Bauer (1889: 252). Rudberg aduce a

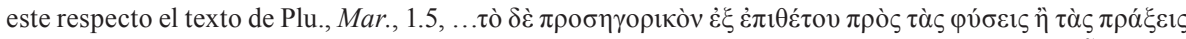

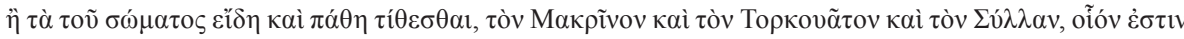

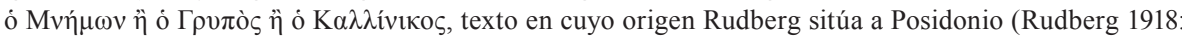
114). Más recientemente puede verse el estudio de Balsdon (1979: 157-158).

${ }^{36}$ Cf. los apuntes de von Fritz (1977: 175). Kidd llega a ser más contundente: "But clearly it was an intelectual discussion, not a practical problem." (Kidd 1988: 910).

${ }^{37}$ En este mismo sentido pueden verse también los apuntes de Malitz (1983: 410).

${ }^{38}$ Ath. 4.36 .151 (= Posidon. T 12). Como bien señala Kidd (1989: 39), los testimonios se refieren a Posidonio como filósofo incluso en contextos referidos a su obra historiográfica (Cf. también Bringmann 1985: 56). Con ello, no creemos que tengamos que hablar de una "filosofía de la historia" como tal desarrollada en la obra de Posidonio - como bien indica Nicolai (2007: 17)-, sino que la filosofía posidoniana avanza sobre el ámbito histórico.

${ }^{39}$ El tratamiento del pasaje por estudiosos de Plutarco deja ver claramente que el pasaje introductorio es interpretado como perteneciente a Plutarco (e. g. Pinheiro 2009: 364; Nikolaidis 2009: 276, n.5). 
positivo sobre Marcelo, que va más allá de lo que realmente el texto posidoniano nos dice. $\mathrm{Y}$ es que ya Kahrstedt advirtió del fuerte tono liviano de la sección introductoria (Kahrstedt 1913: 273), con lo que la segmentación del texto tenía que realizarse de una manera diferente a la planteada con posterioridad por Jacoby, quien, con el mantenimiento del tipo de letra estable en su edición del texto, reconoce y da carta de naturaleza a la totalidad del fragmento como de origen posidoniano, ${ }^{40}$ cosa que la lectura atenta del texto pone en seria duda, máxime a partir de los datos que se han derivado de nuestra revisión de los restantes fragmentos. ${ }^{41}$

La factura general de todo el fragmento delata una difícil continuidad entre las afirmaciones que dan inicio al pasaje, con referencias a Marcelo y su perfil moral, y la narración de la huida de Nicias, quien es el personaje central de una historia que tendría que versar sobre las virtudes romanas de Marcelo. Esta dificultad, señalada como decimos por la crítica ${ }^{42}$ se veía mitigada por parte de los estudiosos dada la necesidad de articular el supuesto excurso sobre Marcelo en la obra de Posidonio. Dale, en su comentario a la vida de Marcelo, ahondaba en esta teoría al apoyarse en una idea presentada por Klotz para dar una ubicación a este fragmento en la obra de Posidonio, en caso de no admitir la interpretación de Jacoby. Para Dale, este fragmento formaría parte de una sección de la obra de Posidonio en la que se hablaba de la relación entre romanos y griegos tomando como marco la captura de Siracusa (Dale 1992: 274), modificando sustancialmente lo que Klotz había propuesto. ${ }^{43}$ Sin embargo, esta deducción tiene pocos puntos de apoyo - cosa que planea por las líneas de Dale-, máxime cuando propone como texto de apoyo un pasaje de Tito Livio inspirado en esa

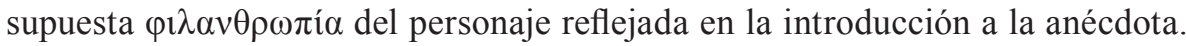
El problema es que cita el pasaje privado de su final, poco favorable a la figura de Marcelo, como ya señaló Carawan, con lo que la visión entre neutra y positiva que tiene Marcelo en la historia de Nicias tendría difícil ajuste con este pasaje ${ }^{44}$ y volveríamos, al caer la captura de Siracusa fuera del marco cronológico

\footnotetext{
${ }^{40}$ Algo que todavía se puede observar en la edición de Vimercati (2004: 678), quien habla de una fuente favorable a Marcelo como núcleo de la narración plutarquea y posidoniana en este pasaje.

${ }^{41}$ Mühl, discípulo de Jacoby y defensor de la existencia de esa monografía sobre Marcelo, es quizá el ejemplo extremo de ese abuso del fragmento. Considera que estamos ante un "wörtliches Excerpt" en el que se incluiría la explicación inicial de Plutarco, y ello da pie a determinar un patrón narrativo que se aplica a los restantes pasajes de la vida plutarquea, en busca de los restos de esa monografía (Mühl 1925: 9). Más recientemente Crawford consideró que el razonamiento plutarqueo respecto a la escena era también debido a Posidonio (Crawford 1978: 197).

42 "The story is firmly centred on Nicias, not on Marcellus, which is a main reason for thinking that 1-10 is Plutarch" (Kidd 1988: 899). Kidd había defendido que 2-9 eran obra de Posidonio por razones de estilo en la página anterior, lo que pone en seria duda la continuidad del pasaje. Vid. también las dudas de Clarke (1999: 180-181).

43 "Die Vermutung liegt nahe, dass er von dem Verhalten des Marcellus gegenüber den Griechen in einer Einleitung zum sizilischen Sklavenkrieg gesprochen habe, wo er die inneren Ursachen des Aufruhrs zu erfassen suchte." (Klotz 1934: 294).

${ }^{44}$ Citamos el pasaje de Tito Livio 25.40.1-2 entero: dum haec in Hispania geruntur, Marcellus captis Syracusis, cum cetera in Sicilia tanta fide atque integritate composuisset ut non modo suam gloriam
} 
de las Historias, al excurso, con el agravante de añadir escenas hasta entonces no planteadas.

Simplemente, creemos que la propuesta de Kahrstedt, sostenida con posterioridad por Klotz y también por Kidd (1988: 345-346 y 899), ha de ser recuperada en todos sus términos, y editar como posidoniano el núcleo de la historia de Nicias, que necesita de una ubicación nueva, fuera de ese marco trazado por Plutarco y que la convertía de manera algo forzada en una narración perteneciente al tratamiento de Marcelo. ${ }^{45}$ El colorista estilo de la narración, en la que seguramente se filtran muchos rasgos estilísticos del propio Posidonio (Kidd 1988: 897-898), nos lleva a pensar en su inclusión en la obra historiográfica y no en el tratado sobre mántica (Malitz 1983: 363, n. 33), aunque resulte difícil desechar esta idea por completo. Si hemos de apostar por una ubicación en la obra histórica, su aparición, como ya señalaba Klotz (1934: 295), en el ámbito de la revuelta siciliana es seguramente la mejor opción: no se trataría, seguramente, de un texto referido a Marcelo, sino que la única razón para colocar en una posición privilegiada a Marcelo en el mismo se debería a la reutilización del mismo por parte de Plutarco.

Con todo ello, los indicios que llevaban a la posible existencia de un excurso centrado en la figura de Marcelo desaparecen por completo, al tiempo que la supuesta visión laudatoria de su figura queda en entredicho. Los cuatro fragmentos que favorecían la existencia de ese núcleo en la obra posidoniana tienen una ubicación justificada en otra sección de la narración, lo que solventa su aparente posición fuera del marco cronológico de la obra. Se trata, en definitiva, de pasajes en los que Posidonio hace referencia a Marcelo pero de manera tangencial, sin que el objeto primero sea centrar la atención en la figura de Marcelo. De este modo, el supuesto excurso sobre Marcelo pasa a descomponerse en dos segmentos: uno, que quizá ni siquiera tendría como protagonista a Marcelo, ubicado en el comienzo de la revuelta de los esclavos en Sicilia, y otro en el excurso sobre el sistema onomástico latino, con lo que Marcelo estaría tratado en un excurso, pero no centrado en su figura. Esto permitiría explicar el espejismo con el que la crítica se tuvo que enfrentar, y que justifica en gran parte la inercia que afecta a la interpretación de la figura de Marcelo. Que los fragmentos procedan de un único núcleo de las Historias de Posidonio es la clave que permite a la crítica ver una imagen más o menos clara de Marcelo, una paradoja difícil de explicar si las referencias a Marcelo eran simplemente apuntes dispersos por la obra del de Apamea.

sed etiam maiestatem populi Romani augeret, ornamenta urbis, signa tabulasque quibus abundabant Syracusae, Romam deuexit, hostium quidem illa spolia et parta belli iure; ceterum inde primum initium mirandi Graecarum artium opera licentiaeque hinc sacra profanaque omnia uolgo spoliandi factum est, quae postremo in Romanos deos, templum id ipsum primum quod a Marcello eximie ornatum est, uertit. Dale cita exactamente hasta augeret. Cf. además las indicaciones de Carawan (1985: 136). Ferrary alertaba sobre lo extraordinario de este primer caso de expolio a los vencidos (Ferrary 1988: 573).

${ }^{45}$ Theiler hablaba de este fragmento como un ejemplo de la filantropía de Marcelo que, como hemos visto, es una caracterización debida al propio Plutarco (Theiler 1982: 90). 


\section{Posidonio y LA COMPREnSión de LA Figura de MARCElo}

A pesar de esta visión ligeramente distante de Marcelo que, según nuestra interpretación, se desprende de los fragmentos, cabe todavía tratar de dar respuesta a la pregunta planteada por Kidd respecto a la posible reescritura por parte de Posidonio de la versión negativa que la historiografía tenía de la figura de Marcelo. Esta ha sido una idea que ha sido mantenida sin fisuras por la crítica, incluso una vez superada la idea del excurso. ${ }^{46}$ Obviamente, las implicaciones de esa visión negativa o positiva serán mucho más difusas al no encontrarnos ante un excurso centrado en la figura de Marcelo, hecho que a nuestro entender no impide que podamos tratar de atisbar la posible imagen que Posidonio tenía de Marcelo, una visión que puede deparar algunas matizaciones en la interpretación general de la relación de Posidonio con el mundo romano.

Aunque es poco el material con el que contamos, la profundidad de los razonamientos que se esconden detrás de la indagación sobre los cognomina por parte de Posidonio nos permite avanzar con paso seguro. Lo primero que llama la atención del lector es el fuerte tono homérico de todos los pasajes: Posidonio se centra en una descripción de tono guerrero, pero además mide bien hasta los términos empleados para llevarnos de manera intencionada a un escenario homérico. Sabemos, gracias al estudio continuado de los fragmentos etnográficos, la importancia que Posidonio otorgaba al universo homérico a la hora de realizar caracterizaciones antropológicas. ${ }^{47}$ Como buen estoico, el trato asiduo con los textos homéricos creó en la mente de Posidonio un universo hacia el cual convergían muchas de las interpretaciones que el de Apamea daba a la hora de valorar un uso o costumbre nuevo o ajeno. Esta suerte de Interpretatio graeca, tan antigua como Heródoto, puede permitirnos recorrer el camino interpretativo que llevó a Posidonio a hacer de Marcelo un nuevo héroe homérico y comprender el porqué de todo ello. Giurovich insistió recientemente en que el $\tilde{\eta} \theta$ o $\varsigma$ de un pueblo y -añadimos nosotros- el de los individuos que lo componen, determina un curso de los acontecimientos, perfilado y observado bajo el marco que ofrece esa interpretación (Giurovich 2005: 36-40).

Sin partir de una visión apriorística que hace de Posidonio el depositario de la información de primera mano que le pudiese tributar la familia de los Marce$10 s,{ }^{48}$ los datos básicos a disposición del de Apamea -y en esto podemos seguir el

${ }^{46}$ Cf. supra, nota 16 , y recientemente, aunque sin análisis profundo de los textos, el listado que Fowler propone (Fowler 2003: 45).

${ }^{47}$ Cf. en términos generales las indicaciones de Pohlenz (2005: 424). Norden por su parte hablaba del paralelo entre la hospitalidad germana y el mundo homérico (Norden 1959: 130-142). Martin ahondaba en la reutilización de léxico homérico en la etnografía céltica (Martin 2002: 579-580). En este mismo sentido, puede verse también el trabajo de Ruggeri (2000: 74-75). Desde una perspectiva quizá menos literaria pueden verse los apuntes sobre lo céltico de Gómez Espelosín, que incide en los aspectos históricos que subyacen a la caracterización estereotipada que las fuentes clásicas -entre ellas Posidonio-nos presentan de estos pueblos (Gómez Espelosín 2004: 211-235).

${ }^{48}$ Como sugerían e. g. Jacoby (1963: 190) y Münzer en su reseña a la obra de Mühl (Münzer 1925: 98-99). 
testimonio de las fuentes y materiales previos que recopiló Fowler- pueden sintetizarse de la siguiente manera: un número de magistraturas muy elevado, una actividad bélica amplísima, un arrojo militar que roza lo imprudente, y la dedicación de los Spolia opima y de un templo a Honos y Virtus.$^{49}$ Con este punto de partida, absoluta e intencionadamente simplista, creemos que puede recorrerse el camino que trazó Posidonio.

Los rasgos en abstracto y su intento de ubicación dentro del mundo griego dan como resultado un diseño de la figura de Marcelo bajo supuestos homéricos. El arrojo ciego e imprudente que condenara Polibio se muestra en Posidonio bajo un análisis psicológico y dentro de la esfera del concepto homérico de $\theta v \mu$ ó ese furor ciego propio de los héroes que marchan al combate. Con ello, Posidonio nos da una interpretación de la visión de la personalidad de Marcelo que curiosamente parte del mismo acercamiento que tenemos en Polibio, pero con un cambio de la óptica que hace que esa interpretación no tenga por qué ser negativa, al menos a primera vista. La sistematicidad de ese empuje bélico se ve además sancionada en la famosa denominación de Marcelo como gípos (F42a-42b): Bake alertaba sobre el paralelo que supone un pasaje plutarqueo en el que se nos dice que Escipión el Africano decía a un joven pertrechado con un escudo lo si-

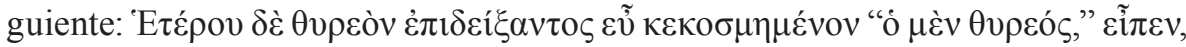

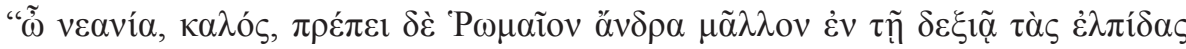

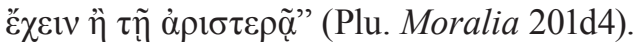

Gracias al estudio del universo que rodea a los Spolia opima debido a Flower, hoy tenemos datos que permiten fundamentar el halo de idealización y de intencionalidad política que rodea a esta ancestral práctica, en la que Marcelo desempeña un papel fundamental (Flower 2000: 34-64). El espíritu que mueve esta práctica es de todo punto agonal: estamos ante la ofrenda de las armas del jefe contrario, dentro de un escenario de luchas individuales que tiene mucho de heroico, y seguramente de ajeno al universo bélico de una época tan avanzada (Flower 2000: 34-36). Más allá de los detalles concretos de esta práctica, lo importante es comprender el sentido que podría tener a ojos de un griego versado en Homero como era Posidonio. El binomio compuesto por el armamento del contrario como trofeo y el carácter sacro de su ofrenda entra claramente dentro de la esfera idealizada del mundo de los poemas homéricos, como ocurre también con el templo consagrado de manera póstuma a Honos y Virtus por el propio Marcelo. ${ }^{50}$ Hasta esta combinación tiene su resabio homérico si por un momento

\footnotetext{
${ }^{49}$ Recuérdese que, en tiempos de Posidonio, la figura de Marcelo tenía una interpretación claramente negativa, que solo sería rehabilitada en época de Tito Livio (Flower 2003: 45), de modo que el factor polémico a la hora de tratar el personaje es algo que Posidonio tuvo que tener presente.

${ }^{50}$ Cf. Liv. 27.25.7; Cic., Verr. 4.121; como bien indica Gros, la idealización llevada a cabo por Cicerón de la figura de Marcelo ha de entenderse en el contexto del discurso (Gros 1979: 89). La vacilación de Tito Livio, que en 29.11.13-14 habla solamente de Aedes Virtutis, no creemos que deba ser llevada más allá de un simple lapsus (Latte 1960: 200).
} 
tratamos de pensar en sus equivalentes griegos, que pueden ser $\tau 1 \mu \eta ́$ y $\dot{\alpha} \rho \varepsilon \tau \eta \dot{~} .^{51}$ Ambos conceptos son fundamentales en el imaginario homérico, simbolizando respectivamente el reconocimiento material de las acciones del héroe y la naturaleza excepcional del mismo. Si cabe alguna duda respecto a esta interpretación, que entra en contradicción con el valor tradicional que Grimal (1975: 122) atribuía a estos dos valores, puede recordarse qué se custodiaba dentro de ese templo. Como nos dice Cicerón, este templo estaba adornado con las estatuas traídas por el propio Marcelo de su andadura en Siracusa. ${ }^{52}$

In hac partitione ornatus non plus uictoria Marcelli populo Romano adpetiuit quam humanitas Syracusanis reseruauit. Romam quae adportata sunt, ad aedem Honoris et Virtutis itemque aliis in locis uidemus.

En definitiva, el hermanamiento de los conceptos divinizados de Honos y Virtus es algo que tiene un encaje incómodo en el mundo romano, y un recorrido ciertamente limitado. ${ }^{53}$ Es interesante subrayar que estamos, como nos recuerda Dumézil, ante una readaptación del templo dedicado por Fabio Cunctator en el 233 con motivo de la victoria sobre los ligures (Dumézil 1966: 388), ${ }^{54}$ una readaptación que además planteaba no pocos problemas de índole religiosa. ${ }^{55}$

Todos estos indicios nos permiten aventurar un núcleo de interpretación de la figura de Marcelo en la obra de Posidonio que parece tomar su punto de partida en el universo del héroe homérico. Esta interpretación, que como venimos diciendo, no es ajena al mundo espiritual de Posidonio, tiene difícil anclaje en la tradición historiográfica de cuño latino e incluso en otro autor griego como es Polibio. Sin embargo, no se debe desechar a la ligera la idea de que esta visión, que emana de los pasajes posidonianos, pueda tener su origen en el filohelenismo tan marcado de la época de Marcelo, ${ }^{56}$ y que se observa tanto en la unión de Honos y

${ }^{51}$ En este sentido, es curioso contemplar el tono en el que Münzer termina su exposición de la vida de Marcelo: "Virtus und Honos waren seine Götter, und die Geschichte seines Lebens ist die seiner Feldzüge." (Münzer 1899: 2754).

${ }^{52}$ Cic. Verr. 4.121. Cf. para los restantes pasajes el artículo de Münzer (1899: 2749).

${ }^{53}$ Será Mario quien, como nos cuenta Cic. Sest., 116, recuperará esta efímera unión de Honos y Virtus. Cf. además las indicaciones de Dumézil (1966: 389-390). Para la poca rigurosidad de Marcelo en lo que a la religión tradicional romana se refiere, baste recordar la afirmación de Cic. N.D. 2.77, Nam ex acuminibus quidem, quod totum auspicium militare est, iam M. Marcellus ille quinquiens consul totum omisit, idem imperator, idem augur optumus. Gros ve en este templo una exaltación del valor individual frente a lo colectivo, lo que encaja con nuestra propuesta (Gros 1979: 106-107).

${ }^{54}$ Cf. Cic. N.D. 2.61.

${ }^{55}$ Dumézil nos recuerda el problema que la doble cella suponía para la manifestación de las divinidades (Dumézil 1966: 389). Los detalles relativos a los testimonios antiguos pueden verse una vez más en el artículo de Münzer (1899: 2753).

${ }^{56}$ Se comprenden así las dudas de Gros a la hora de atribuir a Posidonio el origen de la versión favorable del expolio de Siracusa (Gros 1979: 99-100): parece difícil sostener esta idea sin aceptar la existencia de una obra completa dedicada a Marcelo. Ferrary es mucho más optimista con la posible existencia de una obra dedicada en exclusiva al personaje (Ferrary 1988: 575, n. 9). 
Virtus como en el aliento heleno que se respira en la representación de los Spolia opima,${ }^{57}$ una visión que no triunfará en la historiografía romana. ${ }^{58}$

Con todo ello, y si hemos de optar por una interpretación, creemos que Posidonio habría actuado quizá con cierta simpatía por la figura de Marcelo, pero que la visión que nos ofrece no bebía de las fuentes que la familia de los Marcelos pudieran proporcionarle. En todo caso, y si en algún contexto pudiera tener rendimiento esa visión "helenizada" de Marcelo, hemos de pensar en Rodas, en el ámbito del que provenía el epigrama y el propio Posidonio. De esta manera, el historiador habría actuado con cierta independencia a la hora de generar su interpretación de la figura del héroe venido a menos en Roma, y habría recuperado la que seguramente sería la versión positiva en tono helenizado que de la vida de Marcelo se tendría en ámbitos orientales, dándole una pátina de unidad bajo los postulados propios del universo posidoniano: ese tono homérico se ajustaría bien con el empleado en el análisis del pasado de tintes míticos que vemos aplicado a otras culturas en las Historias. Se trataría, por tanto, de una visión muy particular, por completo alejada de una supuesta maniobra propagandística debida a los descendientes de Marcelo para restaurar su imagen, precisamente por la poca y limitada efectividad que esta visión tendría para contrarrestar la versión negativa de Marcelo en ámbito romano.

A modo de conclusión, la tentativa de reconstrucción de los fragmentos relativos a Marcelo nos permite tomar algunas prevenciones en lo que al manejo de las ruinas posidonianas se refiere. En primer lugar, la imagen que presentara Momigliano de un Posidonio que no se sentía ajeno a nada romano ha de ser perfilada para entenderla de un modo que no haga de Posidonio un perfecto romano en todos los sentidos (Momigliano 1984: 232). Uno de los silencios respecto a las Historias de Posidonio que, por extraño, ha pasado desapercibido a la crítica es el de Cicerón: el Arpinate no menciona en ocasión alguna a Posidonio en su faceta de historiador ${ }^{59}$ salvo en un caso muy conocido pero no por ello menos interesante, el relativo al commentarius sobre su consulado. Sabemos que Cicerón redactó unas notas en griego que hizo llegar a Posidonio para que les diera naturaleza literaria -cosa que el de Apamea rechazó-, y también tenemos

\footnotetext{
${ }^{57}$ Como recuerda Picard “... le rite le plus vénérable n’était pas moins hellénisé dans la forme: la monnaie du milieu du $1^{\text {er }}$ siècle av. J.-C. qui représente Marcellus consacrant ses spolia, et qui dérive sans doute d'une peinture plus ancienne, montre l'imperator chargé d'un petit trophée de forme tout à fait classique." (Picard 1957: 139).

${ }^{58}$ Así, de las dos posibles opciones que planteaba Earl respecto a la ubicación de Posidonio en Roma, o que se adaptara a la Urbs o que llevara una existencia desarrollada en círculos muy reducidos, a nuestro entender, y refiriéndonos a la creación historiográfica, apostamos por el segundo modelo (Earl 1967: 40-41).

${ }^{59}$ Baste remitir a los índices de Kidd para todos los pasajes en que Cicerón habla de Posidonio, unos veintiuno, pocos si atendemos a la importancia del filósofo para tratados como el de De divinatione o De officiis (Kidd 1972: 258-259).
} 
la seguridad de que existía un escrito equivalente en latín. ${ }^{60}$ Con ello, más allá de intentar adentrarnos en un debate estéril sobre los motivos que llevaron al rechazo del encargo por parte de Posidonio, lo que podemos es verificar el funcionamiento a dos niveles muy distintos todavía de la historiografía griega y latina. El pulso marcadamente retórico del vं ó$_{\mu \nu \eta \mu \alpha}$ señalado por el propio Cicerón ${ }^{61}$ nos lleva a un tono historiográfico semejante al visto en la obra de Posidonio, y obliga a pensar que, para el público griego, las expectativas historiográficas se movían todavía en un nivel de tono helenístico. Todo parece apuntar a que el objetivo de Cicerón era difundir sus hazañas en el mundo griego bajo los moldes que ese público requería, y que no encajaban con los que poco a poco iban tomando forma en Roma. Con todo ello, hemos de tener presente que la comprensión de la realidad que Roma ponía ante los ojos de Posidonio se mueve todavía dentro de los parámetros tradicionales de la literatura griega, sumando las impresiones de ese contacto con Roma a los viejos moldes de interpretación de la historiografía griega. Posidonio es un historiador griego que contempla Roma desde la perspectiva de un griego: todavía estamos mucho más cerca en todos los aspectos de la historiografía helenística que de la de época augústea, y ese es precisamente el punto de partida que nos permite separar a Posidonio de Plutarco. ${ }^{62}$ Así, en un excurso sobre los cognomina, Posidonio habría tratado la figura de Marcelo como un personaje que, atendiendo a su carácter temerario, y a su vinculación con los Spolia opima, con Honos y Virtus, haría honor al calificativo de àpíıs, de una manera comprensible a un lector griego, dado el sentido homérico que Posidonio le otorgaba.

La presencia de perspectivas favorables a los optimates en pasajes de Diodoro fue, sin ir más lejos, uno de los indicios claros para atribuir pasajes del siciliano a Posidonio. ${ }^{63}$ No son pocas las dudas que la crítica ha manifestado a este respecto, ${ }^{64} \mathrm{y}$ creemos que la interpretación que ofrecemos de la figura de Marcelo puede, reconociendo su carácter polémico, al menos advertir al estudioso de

${ }^{60}$ Los pasajes son Cic. Att. 1.20 .6 y 2.1.1-2. Para los detalles puede verse el reciente estudio de Riggsby (2007: 271-272). Creemos que la postura de Crawford, quien, al analizar este escrito ciceroniano, habla de cierto "desprecio" de los romanos hacia su literatura, ha de ser suavizada (Crawford 1978: 200-201): Crawford no tiene en cuenta que existía una versión latina del escrito sobre el consulado de Cicerón, lo que apunta la existencia de dos públicos, uno griego y uno romano, y no a esa inferioridad de la literatura latina.

${ }^{61}$ A este respecto pueden verse los apuntes de Nicolai (1992: 112), y Marincola (1997: 182).

62 Pohlenz y Kidd resaltaban que los aspectos estilísticos también apuntaban a ese universo (Pohlenz 2005: 427; Kidd 1989: 43-44). Thümmel hablaba de las Historias como de la última obra "peripatética" tanto en contenido como en forma (Thümmel 1984: 561).

${ }^{63}$ En este sentido, recuérdese, e. g., el importante estudio de Strasburger sobre Posidonio y el imperialismo romano (Strasburger 1982: 921-945)

${ }^{64}$ Contundente es la postura de Raskolnikoff y Botteri, quizá demasiado escéptica y también arriesgada al proponer una fuente latina como sustituta de Posidonio en los correspondientes pasajes diodoreos (Raskolnikoff y Botteri 1979: 135-157). Pelling se pronunciaba de manera más prudente en lo que se refiere a la reconstrucción de Malitz, que abusa de la obra diodorea como punto de apoyo para la reconstrucción de la obra de Posidonio (Pelling 2007: 251). 
que el manejo de estos fragmentos ha de realizarse sin apriorismos que den lugar a reconstrucciones idealizadas, y que las revisiones de los fragmentos en su vertiente más puramente material han de verse acompañadas de la correspondiente reinterpretación del contenido y el pensamiento que transmiten.

\section{BiBLIOGRAFÍA}

Bake 1810: J. Bake, Posidonii Rhodii reliquiae doctrinae. Collegit atque illustravit J. Bake, accedit D. Wyttenbachii annotatio (Leiden 1810).

Balsdon 1979: J. P. V. D. Balsdon, Romans and aliens (London 1979).

Bauer 1889: A. Bauer, "Poseidonios und Plutarch über die römischen Eigennamen", Philologus 47 (1889) 242-273.

Becchi 2009: F. Becchi, "La notion de philanthropia chez Plutarque: contexte social et sources philosophiques", en J. Ribeiro Ferreira, D. Leão, M. Tröster, y P. Barata Dias (eds.) Symposion and Philanthropia in Plutarch (Coimbra 2009) 263-274.

Bringmann 1986: K. Bringmann, "Geschichte und Psychologie bei Poseidonios" en H. Flashar y O. Gigon (eds.) Aspects de la philosophie hellénistique, Entretiens de la Fondation Hardt 32 (Genève 1986) 29-59.

Buttmann 1861: P. Buttmann, Lexilogus, or a critical examination or the meaning and etymology of numerous Greek words and passages, intended principally for Homer and Hesiod (London 1861) (= Berlin 1825).

Caltabiano 1975: M. Caltabiano, "La morte del console Marcelo nella tradizione storiografica”, en M. Sordi (ed.), Storia e propaganda (Milano 1975) 65-81.

Candau 1985: J. M. Candau, "Posidonio y la historia universal", Habis 16 (1985) 107-127.

Carawan 1985: E. Carawan, "The tragic history of Marcellus and Livy's characterization", CJ 80 (1985) 131-141.

Clark 1992: E. D. Clark, A Historical commentary on Plutarch's Marcellus (University of British Columbia 1992).

Clarke 1999: K. Clarke, Between Geography and History. Hellenistic constructions of the Roman World (Oxford 1999).

Crawford 1978: M. H. Crawford, "Greek intellectuals and the Roman aristocracy in the first century B. C.", en P. D. A. Garnsey y C. R. Whittaker (eds.) Imperialism in the Ancient World (Cambridge 1978) 193-208.

Dumézil 1966: G. Dumézil, La religion romaine archaïque (Paris 1966).

Earl 1967: D. Earl, The Moral and Political Tradition of Rome (London 1967).

Ebeling 1963: H. Ebeling, Lexicon Homericum, edidit H. Ebeling (Hildesheim, 1963) (= Leipzig, 1880-1885).

Edelstein 1936: L. Edelstein, “The Philosophical System of Posidonius”, AJPh 57 (1936) 286-325.

Ferrary 1988: J. L. Ferrary, Philhellénisme et impérialisme. Aspects idéologiques de la conquête romaine du monde hellénistique (Roma 1988). 
Flacelière y Chambry 1966: R. Flacelière y É. Chambry, Plutarque, Vies, tome IV, Timoléon-Paul Émile, Pélopidas-Marcellus (Paris 1966).

Flower 1996: H. I. Flower, Ancestor masks and aristocratic power in Roman culture (Oxford 1996)

Flower 2000: H. I. Flower, "The tradition of the Spolia opima: M. Claudius Marcellus and Augustus", CA 19 (2000) 34-64.

Flower 2003: H. I Flower, "Memories of Marcellus. History and Memory in Roman Republic culture" en U. Eigler, U. Gotter, N. Luraghi y U. Walter (eds.) Formen Römischer Geschichtsschreibung von den Afängen bis Livius (Darmstadt 2003) 39-52.

von Fritz 1977: K. von Fritz, "Posidonios als Historiker", en Historiographia antiqua. Commentationes lovanienses in honorem $W$. Peremans septuagenarii editae (Louvain 1977) 163-193.

Giurovich 2005: S. Giurovich, "Considerazioni sul procedere etnografico posidoniano. Ethos vs Historia. L'esempio dei Galli Scordisci e dei Galli Tectosagi", RSA 35 (2005) 23-52.

Gómez Espelosín 2004: F. J. Gómez Espelosín, "La imagen de lo céltico en la historiografía grecorromana” en J. M. Candau, F. J. González Ponce y G. Cruz Andreotti (eds.) Historia y mito. El pasado legendario como fuente de autoridad (Málaga 2004) 211-239.

Grimal 1975: P. Grimal, Le siècle des Scipions, Rome et l'hellénisme au temps des guerres puniques (Paris 1975).

Gros 1979: P. Gros, "Les statues de Syracuse et les dieux de Tarente. La clase politique romaine devant l'art grec a la fin du III' siècle avant J.-C", REL 57 (1979) 85-114.

Herrmann 1979: W. Herrmann, Die Historien des Coelius Antipater (Meisenheim am Glan 1979).

Jacoby 1963: F. Jacoby, Die Fragmente der Griechischer Historiker, zweiter Teil, Kommentar zu nr. 64-105 (Leiden 1963) (=Berlin 1926).

Kahrstedt 1913: U. Kahrstedt, Geschichte der Karthager, von 218-146 (Berlin 1913).

Kidd 1972: I. G. Kidd y L. Edelstein, Posidonius, volume I, the fragments, edited by L. Edelstein and I G. Kidd (Cambridge 1972).

Kidd 1988: I. G. Kidd, Posidonius, volume II, the commentary [dos volúmenes de paginación continua] (Cambridge 1988).

Klotz 1934: A. Klotz, "Die Quellen der plutarchischen Lebenbeschreibung des Marcellus", RhM 84 (1934) 289-318.

Latte 1960: K. Latte, Römische Religiongeschichte (München 1960).

Malitz 1983: J. Malitz, Die Historien des Poseidonios (München 1983).

Marincola 1997: J. Marincola, Authority and tradition in Ancient historiography (Cambridge 1997).

Martin 1961: H. Martin, Jr., "The Concept of Philantropia in Plutarch's lives", AJP 82 (1961) 164-175. 
Martin 2002: M. Martin, "Omero come archetipo culturale nell'etnografia celtica di Posidonio d'Apamea" en F. Montanari (ed.) Omero tremila anni dopo. Atti del congreso di Genova 6-8 iuglio 2000 (Roma 2002) 579-623.

Mason 1974: H. J. Mason, Greek terms for Roman institutions. A lexicon and analysis (Toronto 1974).

Mauersberger 1968: A. Mauersberger, Polybios-Lexicon, Band I, fasc. $1(\alpha-\gamma)$ (Berlin 1968).

Momigliano 1984: A. Momigliano, "Polibio, Posidonio y el imperialismo romano, en La historiografía griega (Barcelona 1984) 226-238 (=Atti della Accademia delle Scienze di Torino, 1972-1973).

Mühl 1925: M. Mühl, Poseidonios und der Plutarchische Marcellus. Untersuchungen zu Geschichtsschreibung des Poseidonios von Apameia (Berlin 1925).

Müller 1849: C. Müller, Historicorum graecorum Fragmenta III (Paris 1849).

Münzer 1899: F. Münzer, “M. Claudius Marcellus (220)”, en G. Wissowa y W. Kroll, (eds.), Paulys Realencyclopädie der Classische Altertumswissenschaft, III 2 (Berlin 1899) 2738-2755.

Münzer 1925: F. Münzer, "M. Mühl, Posidonios un der plutarchische Marcellus", Gnomon I (1925) 96-100.

Nicolai 1992: R. Nicolai, La storiografia nell'educazione antica (Pisa 1992).

Nicolai 2007: R. Nicolai, "The place of History in the Ancient World", en J. Marincola (ed.), A Companion to Greek and Roman historiography (Oxford 2007) $13-26$.

Nikolaidis 2009: A. Nikolaidis, "Philanthropia as sociability and Plutarch's unsociable heroes", en J. Ribeiro Ferreira, D. Leão, M. Tröster, y P. Barata Dias (eds.) Symposion and Philanthropia in Plutarch (Coimbra 2009) 275-288.

Norden 1959: E. Norden, Die germanische Urgeschichte in Tacitus Germania (Darmstadt 1959) (= Leipzig 1923).

van Nuffelen 2009: P. van Nuffelen, "The name game: Hellenistic historians and royal epithets," en P. van Nuffelen (ed.), Faces of Hellenism. Studies in the History of the Earsten Mediterranean (4th century BC-5th Century AD) (Louvain 2009) 93-111.

Pelling 1980: C. Pelling, "Plutarch's Adaptation of his Source-Material", JHS C (1980) 127-141.

Pelling 2007: C. Pelling, "The Greek historians of Rome”, en J. Marincola (ed.), A companion to Greek and Roman historiography (Oxford 2007) 244-258.

Peter 1865: H. Peter, Die Quellen Plutarchs in den Biographien der Römer (Halle 1865).

Picard 1957: G. C. Picard, Les trophées romains. Contribution à l'histoire de la Religion et de l'Art triomphal de Rome (Paris 1957).

Pinheiro 2009: J. J. S. Pinheiro, "O sentido de Philanthropia nas biografias de Coriolano, Cícero e Catão de Útica”, en J. Ribeiro Ferreira, D. Leão, M. Tröster, y P. Barata Dias (eds.) Symposion and Philanthropia in Plutarch (Coimbra 2009) 359-368. 
Pohlenz 2005: M. Pohlenz, La Stoa, Storia di un movimiento spirituale (Milano 2005) (=Göttingen 1959).

Raskolnikoff y Botteri 1979: M. Raskolnikoff y P. Botteri, "Posidonius, nom de notre ignorance," QSt 9 (1979) 135-157.

Reinhardt 1921: K. Reinhardt, Poseidonios (München 1921).

Reinhardt 1926: K. Reinhardt, Kosmos und Sympathie: neue Untersuchungen über Poseidonios (München 1926).

Reinhardt 1954: K. Reinhardt, Poseidonios von Apameia, der Rhodier genannt (Stuttgart 1954).

Riggsby 2007: A. Riggsby "Memoir and Autobiography in Republican Rome" en J. Marincola (ed.), A companion to Greek and Roman historiography (Oxford 2007) 266-274.

Rudberg 1918: G. Rudberg, Forschungen zu Poseidonios (Uppsala-Leipzig 1918).

Rugeri 2000: M. Ruggeri, Posidonio e i Celti (Firenze 2000).

de Sanctis 1968: G. de Sanctis, Storia dei Romani, volumen III, L'età delle Guerre Puniche, parte II (Firenze 1968).

Schulze 1991: W. Schulze, Zur Geschichte lateinischer Eigennamen, mit einer Berichtigungsliste zur Neuausgabe von O. Salomies (Zürich 1991).

Strasburger 1982: H. Strasburger, "Poseidonios über die Römerherrschaft", en W. Schmitthenner y R. Zoepffel (eds.), Studien zur Alten Geschichte II (Hildesheim 1982) 920-945 (=JRS 55 [1965] 40-53).

Theiler 1982: W. Theiler, Poseidonios, die Fragmente, herausgegeben von Willi Theiler, II Erläuterungen (Berlin 1982).

Thümmel 1984: H. G. Thümmel, "Posidonius und die Geschichte”, Klio 66 (1984) 558-561.

Toepelmann 1869: V. Toepelmann, De Posidonio Rhodio rerum scriptore (Bonn 1869).

Vimercati 2004: E. Vimercati, Posidonio, testimonianze e frammenti (Milano 2004).

Walbank 1967: F. W. Walbank, A Historical commentary on Polybius II (Oxford 1967).

von Wilamowitz 1955: U. von Wilamowitz, Der Glaube der Hellenen II (Darmstadt 1955).

Ziegler 1935: K. Ziegler "Plutarchstudien”, RhM 76 (1935) 53-64.

Ziegler 1984: Plutarchi Vitae parallelae, recognoverunt Cl. Lindskog et K. Ziegler, Vol. II Fasc. 2, iterum recensuit K. Ziegler, editionem correctiorem cum addendis curavit H. Gärtner, (Stuttgardt 1994). 